\title{
Influência de um polímero hidroabsorvente sobre a retenção de água no solo
}

\author{
Rubens A. de Oliveira1 ${ }^{1}$ Lucimar S. Rezende ${ }^{2}$, Mauro A. Martinez ${ }^{3}$ \& Glauco V. Miranda ${ }^{4}$ \\ 1 DEA/UFV. CEP 36571-000, Viçosa, MG. Fone: (31) 3899-2735. E-mail: rubens@ufv.br (Foto) \\ 2 DEA/UFV \\ 3 DEA/UFV. Fone: (31) 3899-1910. E-mail: mmauro@ufv.br \\ ${ }^{4}$ DEA/UFV
}

Protocolo 64 - 16/5/2003 - Aprovado em 6/2/2004

\begin{abstract}
Resumo: Com este trabalho, objetivou-se avaliar a influência da concentração de um polímero hidroabsorvente nas características de retenção de água de dois solos classificados como Latossolo Vermelho-Amarelo, de textura franco-argilo-arenosa, e Argissolo Câmbico Vermelho-Amarelo, de textura argilosa. Os estudos consistiram na obtenção das curvas características de retenção de água desses solos misturados com o polímero hidroabsorvente Terracottem ${ }^{\oplus}$, nas concentrações $0,01,0,05,0,10,0,15$ e 0,20 dag kg-1. A avaliação de alteração das características de retenção de água foi feita com base nos valores de disponibilidade total de água (DTA) no solo e nas umidades correspondentes à capacidade de campo e ao ponto de murchamento permanente. Verificou-se que, para potenciais matriciais superiores a $-1,0 \mathrm{MPa}$, a retenção de água foi maior à medida que se aumentou a concentração do polímero na mistura, para os dois solos estudados. No entanto, para potenciais matriciais inferiores, a retenção de água não foi influenciada pela concentração do produto. O polímero, usado na concentração de 0,2 dag $\mathrm{kg}^{-1}$, aumentou a umidade no potencial matricial de $-0,03 \mathrm{MPa}$ em cerca de $41 \%$ para o solo franco-argilo-arenoso e em $37 \%$ para o solo argiloso, em relação às respectivas testemunhas, com aumento da DTA correspondente a 123 e $135 \%$, respectivamente.
\end{abstract}

Palavras-chave: polímero hidroabsorvente, condicionador de solo, retenção de água

\section{Effect of a hydrogel polymer on the soil water retention}

\begin{abstract}
The objective of this research was to evaluate the effect of the concentration of a hydrogel polymer on the water retention characteristics of a sandy clay loam and a clay soil. Water retention curves were obtained for the two soils and for a mixture of these soils with the Terracottem ${ }^{\circledR}$ at $0.01,0.05,0.10,0.15$ and $0.20 \mathrm{dag} \mathrm{kg}^{-1}$ concentrations. The evaluation of the changes in water retention characteristics was done considering the values of the total water availability (TWA), field capacity and the wilting point. For both soils, there was no effect of the hydrogel polymer concentration on the water retention when the matric potentials were less than -1.0 $\mathrm{MPa}$. However, for matric potentials higher than $-1.0 \mathrm{MPa}$, the water retention increased as the concentration of the hydrogel polymer in the mixture increased. The hydrogel polymer, at 0.20 dag $\mathrm{kg}^{-1}$ concentration, increased the water retention, at the $0.03 \mathrm{MPa}$ matric potential, by $41 \%$ for the sandy clay loam and $37 \%$ for the clay soils, when compared to the soils without hydrogel polymer (control treatment) and increment in the available water retention made the TWA of the two soils to increase by 123 and $135 \%$, respectively.
\end{abstract}

Key words: hydrogel polymer, soil conditioner, water retention

\section{INTRODUÇÃO}

$\mathrm{Na}$ década de 80 foram desenvolvidos vários tipos de polímeros sintéticos com diferentes finalidades, alguns recomendados para a utilização agrícola como condicionadores de solo, devido à sua capacidade de melhorar as propriedades físico-químicas do solo. O uso de condicionadores sintéticos tem contribuído para aumentar a capacidade de retenção de água, reduzindo a freqüência de irrigação e permitindo a utilização mais efetiva dos recursos solo e água, contribuindo para melhorar o rendimento das culturas (Nimah et al., 1983; Wang \& Boogher, 1987).

Nos EUA e na Europa os polímeros sintéticos utilizados na agricultura como condicionadores de solo, têm sido amplamente estudados. Entretanto, a grande maioria dos estudos publicados é realizada com os polímeros aniônicos, que atuam 
estruturando o solo, controlando erosão, melhorando a infiltração de água e ajudando na recuperação de solos salinos (Wallace et al., 1986; Wallace \& Wallace, 1986; Shainberg \& Levy, 1994). Poucos são os estudos realizados com os polímeros hidroabsorventes que têm atuação direta na retenção de água. Esses polímeros não reagem com os constituintes do solo, mas exercem efeito direto, aumentando a retenção de água pelo solo (Nimah, et al., 1983).

Nimah et al. (1983) estudaram o efeito de três condicionadores sintéticos nas propriedades físicas de solos de diferentes texturas, tendo sido utilizados o Hygromull na proporção de $200 \mathrm{~kg} \mathrm{ha}^{-1}$, o Agrosil S e o Agrosil LR, ambos na proporção de 2 t ha $^{-1}$. Em solos arenosos o Hygromull aumentou a disponibilidade de água em torno de $125 \%$, ao passo que, para solos argilosos, este acréscimo ficou entre 25 e $30 \%$. O Agrosil LR teve efeito semelhante ao Hygromull para solo argiloso e não foi eficiente para solo arenoso. O Agrosil S não teve efeito na retenção de água nos solos estudados. O Hygromull é uma uréia formaldeído e Agrosil S e Agrosil LR, hidrossilicatos.

Varennes et al. (1997) estudaram o efeito dos polímeros de poliacrilato de sódio Sanwet 3900 e Sanwet 5000 na disponibilidade de água para plantas cultivadas em um solo arenoso. Constatou-se que a retenção de água no solo, determinada entre - 0,01 e-1,5 MPa, aumentou linearmente com os níveis de polímeros incorporados, sendo que, para a concentração de $0,2 \%$, o acréscimo na água disponível para as plantas foi de três vezes, para ambos os polímeros.

Saidrahman \& Iskandark (1998) estudaram, no Usbekistão, o efeito da aplicação de $50 \mathrm{~kg} \mathrm{ha}^{-1}$ de polímeros de poliacrilamida na produtividade do algodoeiro irrigado por sulcos, obtendo $4.200 \mathrm{~kg} \mathrm{ha}^{-1}$ com uma lâmina de $255 \mathrm{~mm}$ de água, parcelada em duas aplicações. Para a testemunha foram necessários $535 \mathrm{~mm}$ de água aplicados em quatro irrigações, obtendo-se uma produção $15 \%$ menor, mesmo se usando cobertura morta. Durante a condução do experimento choveu $300 \mathrm{~mm}$ e os polímeros armazenaram $50 \%$ dessa água na zona radicular.

No Brasil, alguns polímeros sintéticos hidroabsorventes estão sendo utilizados na produção de frutas, hortaliças e mudas de diversas espécies, bem como na formação de gramados em jardins, campos de futebol e de golfe. No entanto, as informações científicas de seu uso como condicionadores de solo são restritas, sendo necessário se conhecer e quantificar a contribuição advinda da aplicação de polímeros hidroabsorventes na disponibilidade de água, em diferentes tipos de solos. Nesse contexto, o trabalho teve como objetivo avaliar a influência da aplicação de diferentes concentrações de um polímero hidroabsorvente nas características de retenção de água de dois solos de texturas argilosa e franco-argilo-arenosa.

\section{MATERIAL E MÉTODOS}

O experimento foi conduzido no Laboratório de Solo e Água do Departamento de Engenharia Agrícola da Universidade Federal de Viçosa, Viçosa, MG, utilizando-se dois materiais de solo e o polímero hidroabsorvente Terracottem ${ }^{\circledR}$. Um dos materiais de solo, classificado como um Latossolo VermelhoAmarelo de textura franco-argilo-arenosa, foi proveniente do município de João Pinheiro, MG, e o outro, classificado como Argissolo Câmbico Vermelho-Amarelo, de textura argilosa, foi oriundo do município de Viçosa.

As massas específicas foram iguais a 1,27 e 1,33 $\mathrm{g} \mathrm{cm}^{-3}$ para os solos de textura franco-argilo-arenosa e argilosa, respectivamente, sendo determinadas pelo método da proveta (EMBRAPA, 1997). Os materiais de solo foram utilizados na forma de TFSA.

O polímero hidroabsorvente foi utilizado nas concentrações 0,01, 0,05, 0,10, 0,15 e 0,20 dag $\mathrm{kg}^{-1}$, abrangendo a faixa de recomendação do fabricante. Essas concentrações são referentes aos materiais de solo seco. Os tratamentos foram compostos pelo material de solo misturado com o polímero, de acordo com cada uma das concentrações usadas. O produto foi adicionado ao material de solo na concentração estabelecida, homogeneizado e guardado em sacos plásticos fechados.

Devido a pouca quantidade usada em cada tratamento, não houve diferença entre os valores das massas específicas de cada material de solo e as respectivas misturas.

As curvas características de retenção de água no solo foram determinadas para os tratamentos com o polímero e para a testemunha, sendo esta constituída apenas por solo, totalizando seis curvas para cada um dos solos estudados. Para se determinar as curvas características de retenção de água nos solos, utilizou-se o Extrator de Richards, aplicandose as pressões de 0,01, 0,03, 0,1,0,3, 1,0 e 1,5 MPa. Os materiais de solo e as misturas foram colocados em anéis de PVC sobre placas de cerâmica saturadas, permanecendo na condição de saturação durante $24 \mathrm{~h}$, fazendo-se três repetições. A determinação das curvas características seguiu a metodologia recomendada pela EMBRAPA (1997).

A retenção de água foi avaliada com base nos valores de disponibilidade total de água no solo (DTA) e dos níveis de umidade nos potenciais matriciais de - 0,03 e - 1,5 MPa, referentes aos valores de capacidade de campo e ponto de murchamento permanente, respectivamente.

Os valores de disponibilidade total de água (DTA) nos solos estudados foram calculados aplicando-se a seguinte expressão:

$$
\mathrm{DTA}=\frac{\mathrm{Cc}-\mathrm{Pm}}{10} \rho
$$

em que:

DTA - disponibilidade total de água, $\mathrm{mm} \mathrm{cm}^{-1}$

Cc - capacidade de campo, dag $\mathrm{kg}^{-1}$

$\mathrm{Pm}$ - ponto de murchamento permanente, dag $\mathrm{kg}^{-1}$

$\rho \quad$ - massa específica do solo, $\mathrm{g} \mathrm{cm}^{-3}$

A análise de regressão foi aplicada para avaliação do efeito das concentrações do polímero hidroabsorvente sobre as características de retenção de água dos dois tipos de solo, usando-se o programa computacional SAEG - Sistema para Análise Estatística. 


\section{RESULTADOS E DISCUSSÃO}

Na Figura 1 está apresentada as curvas de retenção de água ajustadas aos dados experimentais para os solos de textura franco-argilo-arenosa e argilosa, respectivamente, obtidas para a testemunha e as cinco concentrações do polímero hidroabsorvente. O melhor ajuste foi obtido com uso do modelo potencial. Na Tabela 1, têm-se os valores dos coeficientes dos modelos ajustados para as curvas de retenção de água nos solos de textura franco-argilo-arenosa e argilosa, respectivamente, nas diferentes concentrações do polímero, juntamente com os valores de coeficiente de determinação.
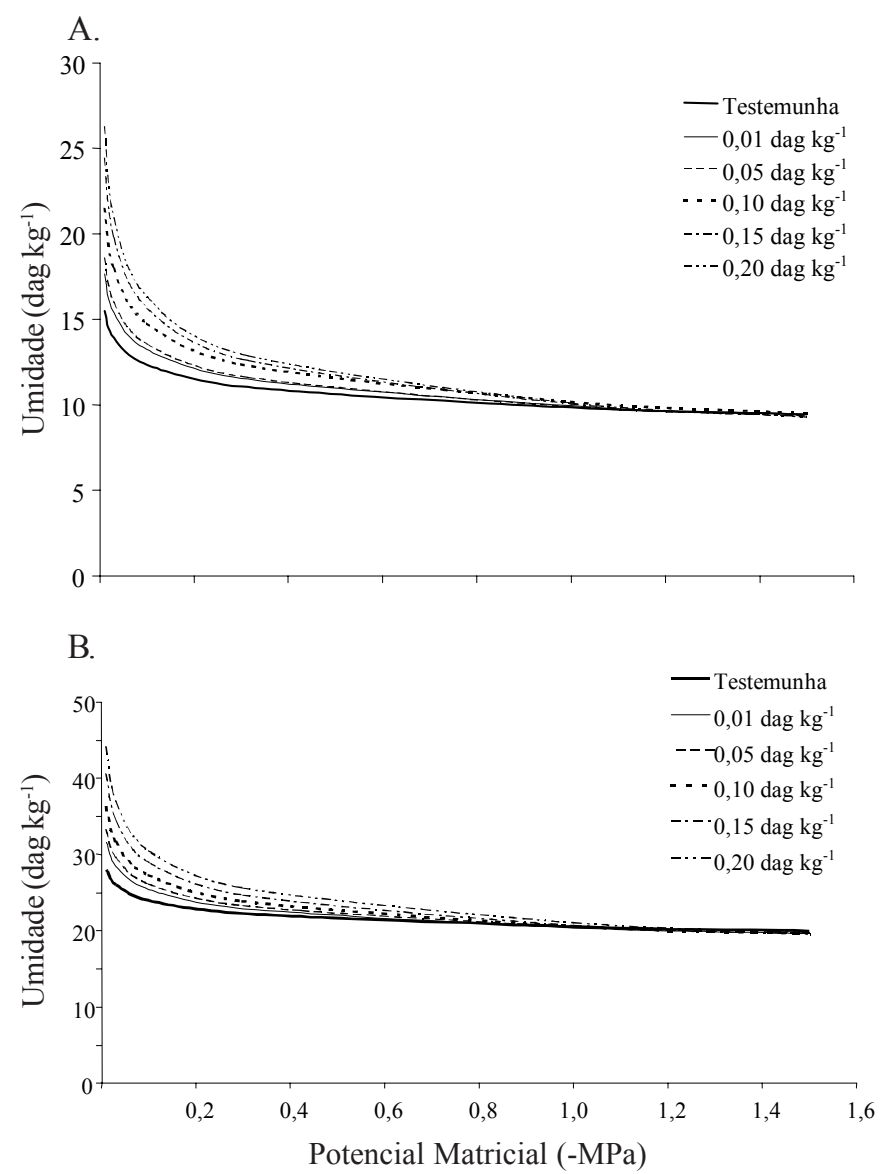

Figura 1. Curvas características de retenção de água no solo de textura (A) franco-argilo-arenosa e (B) argilosa, misturado com o polímero hidroabsorvente, em diferentes concentrações

Como pode ser observado na Figura 1, os dois solos apresentaram o mesmo comportamento, em termos de retenção de água, quando misturados com o polímero hidroabsorvente. À medida que aumentou a concentração do polímero nos solos ocorreu maior retenção de água, principalmente nos potenciais matriciais mais elevados. Em potenciais inferiores a -1,0 MPa para os dois solos, a retenção de água decorrente da adição do polímero não foi significativo, mesmo para a maior concentração estudada, o que pode ser explicado pelo pequeno diâmetro dos grânulos do produto. O polímero apresenta mais de $60 \%$ dos grânulos com diâmetro inferior a 1,0 mm. Rezende (2001) estudou a influência da granulometria do polímero hidroabsorvente Hidratassolo na retenção de água e concluiu que o
Tabela 1. Valores dos coeficientes do modelo potencial ajustado para as curvas de retenção de água no solo de textura (A) franco-argilo-arenosa e (B) argilosa, nas diferentes concentrações do polímero hidroabsorvente, e respectivos valores de coeficiente de determinação $\left(\mathrm{R}^{2}\right)$

\begin{tabular}{crrr}
\hline $\left.\begin{array}{c}\text { Concentração do } \\
\text { Polímero }(\text { dag kg }\end{array}{ }^{-1}\right)$ & \multicolumn{2}{c}{ Coeficientes do Modelo Potencial } & \multirow{2}{*}{$\mathrm{R}^{2}$} \\
\cline { 1 - 3 } A. Franco-argilo-arenosa & Angular & Expoente & \\
0,01 & 9,92 & $-0,1247$ & 0,98 \\
0,05 & 9,88 & $-0,1370$ & 0,98 \\
0,10 & 10,16 & $-0,1620$ & 0,97 \\
0,15 & 10,05 & $-0,1925$ & 0,99 \\
0,20 & 10,12 & $-0,2068$ & 0,98 \\
Testemunha & 9,83 & $-0,0987$ & 0,98 \\
\hline B. Argilosa & & & \\
0,01 & 20,40 & $-0,0953$ & 0,99 \\
0,05 & 20,52 & $-0,1045$ & 0,98 \\
0,10 & 20,58 & $-0,1224$ & 0,98 \\
0,15 & 20,69 & $-0,1459$ & 0,96 \\
0,20 & 21,09 & $-0,1601$ & 0,96 \\
Testemunha & 20,55 & $-0,0675$ & 0,99 \\
\hline
\end{tabular}

diâmetro dos grânulos é de grande importância na retenção de água. Os grânulos de Hidratassolo com diâmetro maior que $2,00 \mathrm{~mm}$ retiveram elevadas quantidades de água em potenciais matriciais de até $-1,5 \mathrm{MPa}$, enquanto em grânulos com diâmetro menor que $0,25 \mathrm{~mm}$ a retenção foi insignificante para potenciais inferiores a $-1,0 \mathrm{MPa}$.

Análises de variância foram feitas com os valores de umidade correspondentes à capacidade de campo $(-0,03 \mathrm{MPa})$ e ao ponto de murchamento permanente (-1,5 MPa), além dos valores de disponibilidade total de água nos dois solos, para os diversos tratamentos.

Na Tabela 2 tem-se as equações de regressão para estimativa dos valores de umidade à capacidade de campo $(\mathrm{Cc})$, ponto de murchamento permanente $(\mathrm{Pm})$ e de disponibilidade total de água no solo (DTA), em função da concentração (C) do polímero, para os solos de textura franco-argilo-arenosa e argilosa.

Tabela 2. Equações de regressão e seus respectivos coeficientes de determinação $\left(\mathrm{R}^{2}\right)$, para estimativa da umidade na capacidade de campo $(\mathrm{Cc})$ e no ponto de murchamento permanente (Pm) e da disponibilidade total de água (DTA) nos solos de textura franco-argilo-arenosa e argilosa, em função da concentração (C) do polímero hidroabsorvente

\begin{tabular}{|c|c|c|c|}
\hline Solo & Variável & Equação & $\mathrm{R}^{2}$ \\
\hline \multirow{3}{*}{$\begin{array}{l}\text { Franco-argilo- } \\
\text { arenoso }\end{array}$} & $\mathrm{Cc}\left(\operatorname{dag~kg}{ }^{-1}\right)$ & $14,46+29,39 \mathrm{C}$ & $0,94 * *$ \\
\hline & $\operatorname{Pm}\left(\right.$ dag kg $\left.{ }^{-1}\right)$ & $9,73+0,20 \mathrm{C}$ & ns \\
\hline & DTA $\left(\mathrm{mm} \mathrm{cm}^{-1}\right)$ & $0,60+3,69 \mathrm{C}$ & $0,94 * *$ \\
\hline \multirow{3}{*}{ Argiloso } & $\mathrm{Cc}\left(\mathrm{dag} \mathrm{kg}^{-1}\right)$ & $27,42+50,31 \mathrm{C}$ & $0,95 * *$ \\
\hline & $\operatorname{Pm}\left(\right.$ dag kg $\left.{ }^{-1}\right)$ & $20,26-2,79 \mathrm{C}+22,12 \mathrm{C}^{2}$ & $0,76^{* *}$ \\
\hline & DTA $(\mathrm{mm} / \mathrm{cm})$ & $0,96+6,50 \mathrm{C}$ & $0,94 * *$ \\
\hline
\end{tabular}

ns-não significativo

** As equações de regressão são significativas a $1 \%$ pelo teste de $\mathrm{F}$

Quando se usou o polímero hidroabsorvente na concentração de $0,2{\text { dag } \mathrm{kg}^{-1}}^{-1}$ o nível de umidade no potencial matricial correspondente à capacidade de campo aumentou em cerca de $41 \%$ para o solo franco-argilo-arenoso, e em $37 \%$ para o solo argiloso, em relação às respectivas testemunhas. No caso do 
solo franco-argilo-arenoso, as concentrações do polímero não afetaram significativamente o nível de umidade no ponto de murchamento permanente, como pode ser observado na Tabela 2. Nesta concentração de polímero a DTA aumentou em $123 \%$ para o solo franco-argilo-arenoso e em $135 \%$ para o solo argiloso.

De modo geral, o polímero foi mais eficiente na retenção de água para o solo argiloso, considerando-se as outras concentrações estudadas. $\mathrm{O}$ comportamento das curvas de retenção dos tratamentos com polímero indicou que o produto pode funcionar como reservatório de água no solo, pelo fato de reter água em elevados potenciais matriciais, impedindo ou reduzindo as perdas por percolação profunda quando houver um aporte de água que ultrapasse sua capacidade de retenção. Saidrahman \& Iskandark (1998) obtiveram conclusões semelhantes quando usaram polímero de poliacrilamida no solo, que resultou em aumento no armazenamento de água de chuva e de irrigação.

\section{CONCLUSÕES}

1. O uso do polímero hidroabsorvente contribuiu para aumentar a retenção de água nos solos de texturas franco-argiloarenosa e argilosa, até o potencial matricial de -1,0 MPa.

2. A medida em que aumentou a concentração do polímero nos solos, ocorreu maior retenção de água, principalmente nos potenciais matriciais mais elevados.

3. O polímero hidroabsorvente, usado na concentração de 0,2 dag $\mathrm{kg}^{-1}$, aumentou a umidade no potencial matricial de $0,03 \mathrm{MPa}$ em cerca de $41 \%$ para o solo franco-argilo-arenoso e em $37 \%$ para o solo argiloso, em relação às respectivas testemunhas, com aumento da DTA correspondente a 123 e $135 \%$, respectivamente.

\section{LITERATURA CITADA}

EMBRAPA. Manual de métodos de análises de solo/Centro Nacional de Pesquisa de Solos. 2. ed. Rio de Janeiro; 1997. 212 p.: il. (EMBRAPA - CNPS. Documentos; 1).

Nimah, N.M.; Ryan, J.; Chaudhry, M.A. Effect of synthetic conditioners on soil water retention, hydraulic conductivity, porosity, and aggregation. Soil Science Society of America Journal, Madison, v.47, p.742-745, 1983.

Rezende, L.S. Efeito da incorporação de polímeros hidroabsorventes na retenção de água de dois solos. Viçosa: UFV, 2001. 74p. Dissertação Mestrado.

Saidrahman, S.M.; Iskandark, K.A. Polymer hidrogel soil additives reduce irrigation requeriments and enhance crop production. In: Inter-Regional Conference on EnvironmentWater: Innovative Issues in Irrigation and Drainage, 1st, 1998, Lisbon, Portugal [Additional Papers, Portuguese National Committee on Irrigation and Drainage],1998. p. 390393.

Shainberg, I.; Levy, G.J. Organic polymers and soil sealing in cultivated soils. Soil Science, Baltimore, v.158, n:4, p.267273, 1994.

Varennes, A. de; Balsinhas, A.; Carqueja, M.J. Effects of two Na polyacrylate polymers on the hydrophysical and chemical propertiers of a sandy soil, and on plant growth and water economy. Revista de Ciências Agrárias, Lisboa, v.4, p.13-27, 1997.

Wallace, A.; Wallace G.A.; Abouzamzam, A.M. Amelioration of soil sodic with polymers. Soil Science, Baltimore, v.141, n:5, p.359-362, 1986.

Wallace, G.A.; Wallace, A. Control of soil erosion by polymeric soil conditioners. Soil Science, Baltimore, v.141, n:5, p.363367, 1986.

Wang, Y.T.; Boogher, C.A. Effect of a medium-incorporatel hydrogel on plant growth and water use of two foliage species. Journal of Environment Horticulture, Washington, v.5, n.3, p.125-127, 1987. 